\title{
Adverse childhood experiences and maternal anxiety and depression: a meta- analysis
}

Nicole Racine ${ }^{1,2}$, Chloe Devereaux ${ }^{1}$, Jessica E. Cooke ${ }^{1,2}$, Rachel Eirich ${ }^{1,2}$, Jenney Zhu ${ }^{1,2}$ and Sheri Madigan ${ }^{1,2^{*}}$

\begin{abstract}
Background: It has been proposed that adverse childhood experiences (ACEs) can put women at risk for mental illness in the pregnancy and postpartum periods. While some studies have found strong support for this proposition, others have found weak or no support. This study is a meta-analysis of the association between ACEs and maternal mental health to resolve between-study discrepancies, and to examine potential moderators of associations.
\end{abstract}

Methods: Three electronic databases (i.e., MEDLINE, Embase, and PsycINFO) were searched up to November 2018 by a health sciences librarian. A hand search was conducted in January 2020 and relevant studies were added. Included studies reported on associations between ACEs and maternal depression and/or anxiety in the perinatal period (pregnancy to 1-year postpartum). Pregnancy and postpartum outcomes were examined separately for both depression and anxiety. Random-effect meta-analyses were conducted. Moderator analyses were conducted using meta-regression. Study quality was evaluated using a 15-point scale.

Results: The initial search yielded 4646 non-duplicate records and full text review occurred for 196 articles. A total of 15 studies $(N=7788)$ were included in the meta-analyses, of which 2 were also described narratively. Publication year ranged from 1998 to 2019. Mothers were approximately 28.93 years of age when they retrospectively reported on their ACEs. All studies had maternal self-report questionnaires for the mental health outcomes. Study quality ranged from 7 to 12. The pooled effect sizes between ACEs and prenatal $(N=12 ; r=.19 ; 95 \% \mathrm{Cl}=.13,24)$ and postpartum $(N=7 ; r=$ $.23 ; 95 \% \mathrm{Cl}=.06$ to .39 ) depressive symptoms were significant. The pooled effect size between ACEs and prenatal anxiety was also significant $(N=5 ; r=.14 ; 95 \% \mathrm{Cl}=.07, .21)$. Moderator analyses indicated that timing of depressive and anxiety symptoms may be important for understanding associations.

Conclusions: ACEs confer risk to maternal mental health, albeit effect sizes are small to moderate in magnitude. Trauma-informed approaches, as well as increased mental health support during and after pregnancy, may help to offset the relative risk of ACEs on maternal mental health.

Keywords: Adverse childhood experiences, Anxiety, Depression, Pregnancy, Postpartum

\footnotetext{
*Correspondence: sheri.madigan@ucalgary.ca

${ }^{1}$ Department of Psychology, Faculty of Arts, University of Calgary, 2500

University Dr. NW., Calgary, AB T2N 1N4, Canada

${ }^{2}$ Alberta Children's Hospital Research Institute, Calgary, AB T2N 1N4, Canada
}

(C) The Author(s). 2021 Open Access This article is licensed under a Creative Commons Attribution 4.0 International License, which permits use, sharing, adaptation, distribution and reproduction in any medium or format, as long as you give appropriate credit to the original author(s) and the source, provide a link to the Creative Commons licence, and indicate if changes were made. The images or other third party material in this article are included in the article's Creative Commons licence, unless indicated otherwise in a credit line to the material. If material is not included in the article's Creative Commons licence and your intended use is not permitted by statutory regulation or exceeds the permitted use, you will need to obtain permission directly from the copyright holder. To view a copy of this licence, visit http://creativecommons.org/licenses/by/4.0/. The Creative Commons Public Domain Dedication waiver (http://creativecommons.org/publicdomain/zero/1.0/) applies to the data made available in this article, unless otherwise stated in a credit line to the data. 


\section{Background}

It is well established that maternal mental health difficulties are common. Approximately $20-30 \%$ of women in low- and middle-income countries and $10 \%$ of women in high-income countries have a mental illness, such as anxiety or depression, during pregnancy or in the postpartum period $[1,2]$. Maternal mental health difficulties are particularly problematic because they put both maternal and child health at risk [3]. For example, pregnant women with mental health difficulties are more likely to have poor health, such as obstetrical complications and preterm labour [4], and to maintain health-risk behaviours such as alcohol and drug use in pregnancy [5]. In addition maternal mental health difficulties are associated with increased risk for poor infant developmental health (i.e., physical, cognitive, socio-emotional) [6-9]. A recent prevalence study in the UK found that anxiety and depression are the most common maternal mental illnesses and that $53 \%$ of children are at risk of being exposed to maternal mental illness prior to age 16 years [10]. Thus, understanding and mitigating the precursors to maternal mental illness in pregnancy and after childbirth are critical for reducing this large-scale public health burden.

Maternal mental health difficulties are strongly associated with social determinants of health such as poverty, devalued social roles, and gender-based violence [11]. Experiences of trauma and violence also put women disproportionately at risk for mental health difficulties in the perinatal period. An emerging body of research has examined how adverse childhood experiences (ACEs), such as abuse, neglect, and household dysfunction experienced prior to the age of 18 , have downstream consequences on mental health in the perinatal period (i.e., pregnancy and postpartum) (e.g., [12, 13]). It has been theorized that experiences of adversity early in life may make an individual more vulnerable to experiencing mental health difficulties in adulthood via alteration to the stress response and affect regulation systems [14], which play a key role in the development of mental health difficulties [15]. Indeed, previous research has demonstrated that the accumulation of ACEs puts women at risk for depressive symptoms both in pregnancy and the postpartum period [12, 13]. As a result of this body of research, there has been a call to implement trauma-informed approaches to perinatal care [16, 17]. To inform trauma-informed approaches to patient care, it is critical to evaluate the state of the science on ACEs and maternal mental health outcomes.

Despite several studies demonstrating an association between exposure to ACEs and poor maternal mental health, there is also research that has found no association between exposure to ACEs and maternal postpartum depression [18]. Given these conflicting findings, the first goal of the current study is to conduct a metaanalysis of the association between ACEs and maternal mental health difficulties in pregnancy and the postpartum period. When study findings are heterogeneous, it often suggests that there are important moderators of the association. That is, there are methodological or social characteristics that may amplify or attenuate associations. For example, previous research suggests there are different clinical profiles of perinatal depression and anxiety based on timing of symptom onset, such that earlier onset of symptoms in pregnancy and postpartum may be indicative of more severe and chronic problems [19]. Thus, examining the timing of perinatal mental health difficulties in relation to maternal ACE exposure may allow for more tailored screening and treatment during the perinatal period [19]. Further methodological differences, such as study quality, may help to explain heterogeneous findings. Additionally, sociodemographic risk (e.g., low income) may moderate the association by exerting an additive risk effect on the link between ACEs and maternal mental health [20,21]. Finally, exposure to an increased number of ACEs may confer a greater risk of clinically severe perinatal depression and/or anxiety [22]. Thus, the second goal of the current study is to identify moderators of the association between ACEs and maternal mental health in order to explain "when" and "for whom" associations are stronger or weaker.

In sum, in order to elucidate health disparities in maternal mental health research, the current meta-analytic study had two aims: 1) provide a pooled effect size of the association between ACEs and maternal mental health difficulties (i.e., anxiety and depression) in pregnancy and the postpartum periods among observational studies, and 2) to examine potential moderators of this association.

\section{Methods \\ Definitions of constructs}

Adverse Childhood Experiences (ACEs) included cumulative retrospective self-reports of child adversity prior to the age of 18 years, including maltreatment and household dysfunction. Measures that were included either consisted of the original 8-item ACEs developed by Felitti and colleagues [23], including physical abuse, sexual abuse, emotional abuse, parent substance use, parent divorce/ separation, parent incarceration, parent mental health problem, and exposure to domestic violence, or the 10item version, which includes the addition of physical neglect and emotional neglect. Prenatal depressive symptoms included depressive symptoms either self-reported (e.g., Center for Epidemiologic Studies Depression Scale) or assessed (e.g., diagnostic codes or diagnosed depressive disorder) during pregnancy. Postpartum depressive symptoms included depressive symptoms either self-reported 
(e.g., Edinburgh Postnatal Depression Scale) or assessed (e.g., diagnosis of depression) after the birth of a child and prior to 12-months postpartum. Prenatal anxiety symptoms included anxiety symptoms either self-reported (e.g., State Trait Anxiety Scale) or assessed (e.g., diagnosed anxiety disorder) during pregnancy. Postpartum anxiety symptoms included those either self-reported (e.g., Generalized Anxiety Disorder, 7-item) or assessed (e.g., diagnosed disorder) after the birth of a child and prior to 12months postpartum. Perinatal depression and perinatal anxiety referred to measurements of anxiety and depression that occurred in both pregnancy and the postpartum period.

\section{Search strategy}

PRISMA guidelines were used for this meta-analysis [24]. The PRISMA checklist is included in Supporting Information 1. Searches were conducted in MEDLINE, Embase, and PsycINFO up to November 2018 by a health sciences librarian. A hand search was conducted in January 2020 and relevant studies were added. This meta-analysis was part of a broader search on adverse childhood experiences (ACEs) and thus outcome terms were not restricted or included as search terms (See Supporting Information 2). Text word fields were searched for the acronym ACEs along with the phrase "adverse childhood event or experiences". Both adjacency operators and truncation symbols were used to capture variations in phrasing. Years were limited from 1998 when the original ACEs study occurred [23]. No language restrictions were applied.

\section{Study inclusion and exclusion criteria}

Titles and abstracts yielded from the search strategy were reviewed by 2 independent coders and evaluated to determine inclusion criteria, which were as follows: (1) ACEs prior to the age of 18 years; (2) a measure of maternal depression or anxiety in pregnancy or prior to 12months postpartum; and (3) statistical data that could be transformed into an effect size. As outlined in Fig. 1, full-length studies were excluded if they (1) did not have a maternal mental health or ACEs measure; (2) were qualitative in nature; (3) did not have a cumulative ACEs measure; (4) were not in English; (5) were from an overlapping sample, or (6) an effect size could not be extracted.

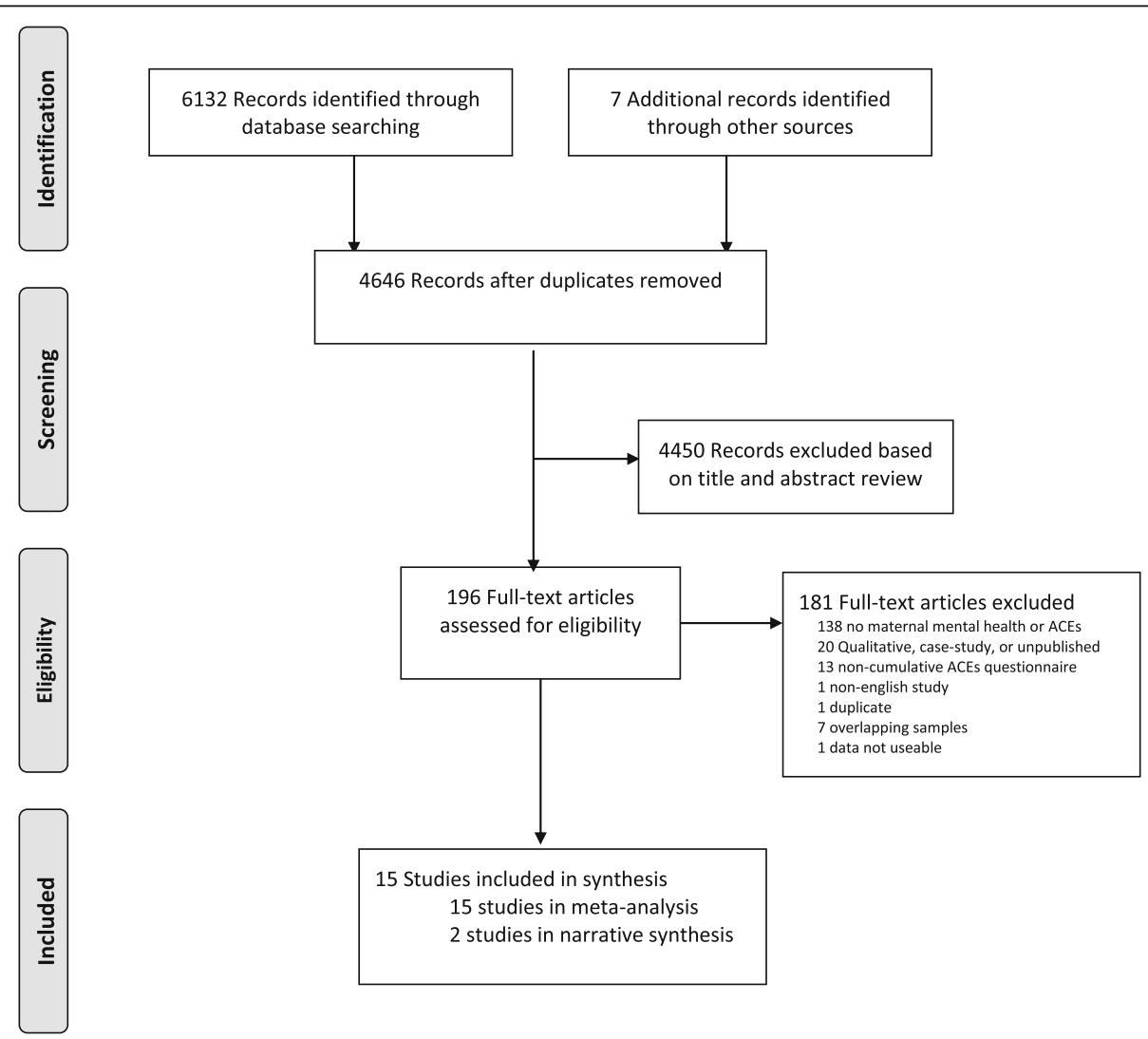

Fig. 1 PRISMA flow diagram detailing the search strategy 


\section{Study quality assessment}

A 15-point scale was used to evaluate the quality of included studies based on a tool adapted from the National Institutes of Health (NIH) Quality Assessment Tool for Observational Cohort and Cross-sectional studies (See Supporting Information 3). Study quality was assessed by one primary coder and $20 \%$ of the ratings were verified by a second coder. The intraclass correlation among coders using a random effects model was .81. Discrepancies were resolved through consensus. All ratings are provided in Supporting Information 4.

\section{Data extraction}

Effect sizes for the association between maternal ACEs and mental health outcomes were extracted. Moderator variables were also extracted including: (1) syndromal level of mental health difficulty (i.e., categorized as $1=$ majority of sample had a diagnosed mental health disorder, $0=$ majority of sample did not have a diagnosed mental health disorder); (2) sociodemographic risk (i.e., 1 = sample had one or more of the following risks: low income, single parents, low education, adolescents, mostly racial and/or ethnic minorities, or pre-existing health difficulties, $0=$ no sociodemographic risk); (3) maternal age at mental health measurement; (4) prenatal (i.e., weeks of pregnancy) or postpartum time (i.e., months post-partum) when outcome measured; and (5) study quality, based on NIH tool described above. All studies meeting inclusion criteria were independently coded by 2 trained coders. Reliability for continuous moderators ranged from 80.00 to $100.00 \%$ agreement and for categorical moderators the mean percentage agreement was $86.70 \%$. Discrepancies were resolved through consensus.

\section{Data synthesis}

Effect sizes, including correlation coefficients, regression coefficients, chi-square values, odds-ratios, and medians were extracted from studies. While the majority of studies reported raw correlation coefficients, all other measures of effect size were converted to correlations for ease of analysis and interpretation. As not all studies adjusted for the same covariates, the use of unadjusted statistics was employed [25].

Only one effect size per study was selected. If there were multiple studies based on the same dataset, the study with the largest sample size and most complete data extraction information was selected. If a study assessed both depression and anxiety, as well as provided different time points of measures (i.e. prenatal and postpartum), all effect sizes were extracted and included in separate meta-analyses. If multiple measurements of anxiety or depression were provided (e.g., Center for Epidemiological Studies Depression Scale and Patient
Health Questionnaire), a pooled effect size was derived and used in the meta-analysis.

\section{Data analysis}

Meta-analyses were conducted when there were more than three studies; otherwise findings were described narratively. Pooled effect sizes and moderator analyses were conducted using Comprehensive Meta-Analysis Software, Version 3.0 [26]. When a study reported nonsignificant findings without an effect size $(n=1)$, a $p$ value of .50 was entered, consistent with recommendations by Rosenthal [27]. Pooled effect sizes are represented as correlations $r$ with $95 \%$ confidence intervals. Using guidelines provided by Funder and Ozer [28], correlations of .10, .20, and .30 were considered small, medium, and large in magnitude, respectively. All calculations were based on random-effects models given the assumption of variation in population parameters among studies [29]. Outliers were examined by investigating whether the confidence intervals of studies overlapped with the overall effect [30]. Only one study did not overlap with the confidence intervals [12]. Sensitivity analyses determined that exclusion of this study did not significantly impact the overall effect size $(r=.199,95 \%$ $\mathrm{CI}=.15, .25)$ and thus the study was retained. Sensitivity analyses based on outliers, attrition and study quality were also conducted.

Heterogeneity of effect sizes was examined using the $Q$ and $I^{2}$ statistics. Examination of moderators is warranted when the $Q$ statistic is significant and/or when the $I^{2}$ statistic is greater than $50 \%$ [29]. Significance of categorical and continuous moderators was determined by the $Q$ statistic and by meta-regressions, respectively [31]. Based on recommendations from Borenstein and colleagues [29], categorical moderators were only explored when more than 3 studies per level of the moderator were available. The Egger test and examination of funnel plots were examined to explore potential publication bias.

\section{Results}

\section{Studies selected}

Details of the process for study selection can be found in the PRISMA diagram (Fig. 1). The initial search yielded 4646 non-duplicate records and full text review occurred for 196 articles. A total of 15 studies are included in the meta-analyses [12, 13, 18, 22, 32-42], of which 2 are also described narratively $[18,37]$. Characteristics of the included studies can be found in Table 1.

\section{Sample characteristics}

Of the studies included in the meta-analyses, the total number of participants was 7788 with samples sizes ranging from 25 to 1994. Publication year ranged from 1998 
Table 1 Characteristics of studies included

\begin{tabular}{|c|c|c|c|c|c|c|c|}
\hline $\begin{array}{l}\text { First Author, } \\
\text { Year, Reference }\end{array}$ & $N^{a}$ & $\begin{array}{l}\text { Mat age at ACEs } \\
\text { measure (years) }\end{array}$ & Country & $\begin{array}{l}\text { Mat mental } \\
\text { health measure }\end{array}$ & $\begin{array}{l}\text { Mat mental } \\
\text { health time point }\end{array}$ & $\begin{array}{l}\text { Name of mat mental } \\
\text { health measure }\end{array}$ & $\begin{array}{l}\text { Type of ACEs } \\
\text { Measure }\end{array}$ \\
\hline Angerud 2018 [22] & 1188 & 30.83 & Sweden & Depression & $\begin{array}{l}\text { Prenatal } \\
\text { Postpartum }\end{array}$ & EPDS & 10-item \\
\hline $\begin{array}{l}\text { Appleton } 2019 \\
\text { [32] }\end{array}$ & 126 & 28.9 & USA & Depression & Prenatal & EPDS & 10-item \\
\hline Folger 2018 [33] & 257 & - & USA & Depression & Postpartum & EPDS & 10-item \\
\hline $\begin{array}{l}\text { Fredriksen } 2017 \\
\text { [34] }\end{array}$ & 762 & 30.3 & Norway & $\begin{array}{l}\text { Depression } \\
\text { Anxiety }\end{array}$ & $\begin{array}{l}\text { Prenatal } \\
\text { Postpartum }\end{array}$ & $\begin{array}{l}\text { EPDS } \\
\text { PRAQ-R }\end{array}$ & 10-item \\
\hline Hantsoo 2019 [35] & 48 & 27.97 & USA & $\begin{array}{l}\text { Depression } \\
\text { Anxiety }\end{array}$ & Prenatal & $\begin{array}{l}\text { EPDS } \\
\text { STAI State } \\
\text { STAI Trait }\end{array}$ & 10-item \\
\hline Howell 2017 [36] & 101 & 26 & USA & Depression & Prenatal & CES-D & 10-item \\
\hline $\begin{array}{l}\text { Letourneau } 2019 \\
\text { [37] }\end{array}$ & 907 & 32.16 & Canada & $\begin{array}{l}\text { Depression } \\
\text { Anxiety }\end{array}$ & $\begin{array}{l}\text { Prenatal } \\
\text { Postpartum }\end{array}$ & $\begin{array}{l}\text { EDS } \\
\text { SCL-90-R- Anxiety }\end{array}$ & 10-item \\
\hline Menke 2018 [18] & $\begin{array}{l}250- \\
\text { prenatal } \\
328- \\
\text { postpartum }\end{array}$ & - & USA & $\begin{array}{l}\text { Depression } \\
\text { Anxiety }\end{array}$ & $\begin{array}{l}\text { Prenatal } \\
\text { Postpartum }\end{array}$ & $\begin{array}{l}\text { EPDS-10 } \\
\text { GAD-7 }\end{array}$ & 10-item \\
\hline Mersky 2018 [38] & 735 & 23.7 & USA & Depression & Prenatal & EPDS & 10-item \\
\hline $\begin{array}{l}\text { Miller-Graff } 2018 \\
\text { [39] }\end{array}$ & 101 & 26 & USA & Depression & Prenatal & CES-D & 10-item \\
\hline Morrison 2017 [40] & 25 & 26.92 & USA & Depression & Postpartum & EPDS & 10-item \\
\hline Narayan 2018 [41] & 101 & 29.1 & USA & Depression & Prenatal & EPDS & 10-item \\
\hline Racine 2020 [12] & 1994 & 33.87 & Canada & Depression & $\begin{array}{l}\text { Prenatal } \\
\text { Postpartum }\end{array}$ & EPDS & 8-item \\
\hline Wajid 2019 [13] & 636 & - & Canada & Depression & Prenatal & EPDS & 10-item \\
\hline $\begin{array}{l}\text { Young-Wolff } 2019 \\
\text { [42] }\end{array}$ & 355 & 30 & USA & $\begin{array}{l}\text { Depression } \\
\text { Anxiety }\end{array}$ & Prenatal & PHD-9 & 8-item \\
\hline
\end{tabular}

CES-D Centre for Epidemiologic Studies Depression Scale, EDS Edinburgh Depression Scale, EPDS Edinburgh Postpartum Depression Scale, GAD-7 Generalized Anxiety Disorder 7-Item, PHQ-9 Patient Health Questionnaire, PRAQ-R Pregnancy-Related Anxiety Questionnaire-Revised, SCL-90-R Symptom Checklist-90, Revised, STAI State State Trait Anxiety Inventory- State, STAI Trait State Trait Anxiety Inventory- Trait. -: value not reported in the manuscript

${ }^{\text {a }}$ Sample size entered into the meta-analysis

to 2020. In all studies, mothers provided a retrospective report of their ACEs. On average, mothers were 28.81 years of age (range 23.70 to 33.87) when they retrospectively reported on their ACEs. The majority of studies used the 10 -item ACEs questionnaire $(n=13)$ with only 2 studies using the 8 -item questionnaire. All studies had maternal report questionnaires for the mental health outcomes. There were a total of $n=12$ studies with prenatal depression outcomes, $n=7$ studies with postpartum depression outcomes, $n=5$ studies with prenatal anxiety outcomes, and $n=2$ studies with postpartum anxiety outcomes. Two studies had depression outcomes measured in pregnancy or the postpartum period (i.e., perinatal depression) and one study had perinatal anxiety outcomes. Only one study included a clinical sample and 6 studies used cross-sectional designs while the other 9 studies had longitudinal designs. Of studies included in the meta-analysis, $66.67 \%(n=10)$ were conducted in the USA, $20.00 \%$ in Europe $(n=3)$, and $13.30 \%$ in Canada $(n=2)$. Study quality ranged from 7 to 12 (Mean=9.07, $\mathrm{SD}=1.5)$. Only one study few within the range of low methodological quality, defined as $1 \mathrm{SD}$ below the mean.

\section{Meta-analytic results for maternal ACEs and prenatal depressive symptoms}

A total of 12 studies (6116 participants) were available for this random-effects meta-analysis, which produced a significant and positive pooled effect size $r=.19$ (95\% CI $=.13, .24$; Fig. 2). Thus, a higher number of maternal ACEs was associated with higher reports of depressive symptoms in pregnancy. The funnel plot inspection revealed asymmetry (Supporting Information 5) and the Egger test was significant, indicating that studies with smaller sample sizes had more extreme values. When results were adjusted by adding 5 imputed studies using Duval and Tweedie's trim and fill technique, the adjusted effect size was $r=.15$ (95\% CI =.09, .20; Fig. 2), but remained statistically significant. Between study heterogeneity was indicated $\left(Q=42.38, p<.001\right.$ and $I^{2}=$ 


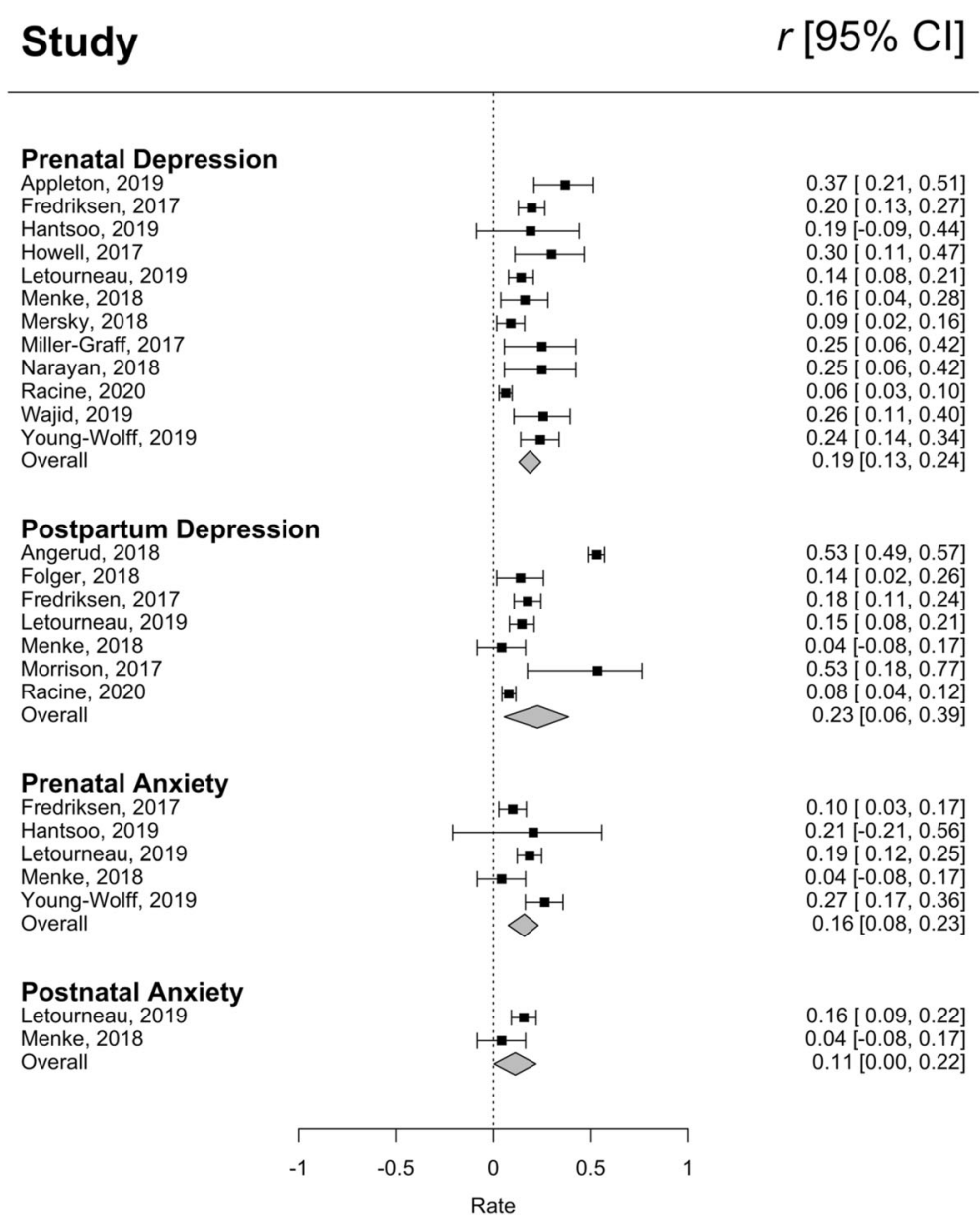

Fig. 2 Forest plot of effect sizes for maternal ACEs and mental health in pregnancy and the postpartum

74.05) and moderators were explored (Supporting Information 6); however, none emerged as significant.

Sensitivity analyses that removed one study that had the highest level of attrition [12] was conducted, however the effect size without this study $(r=.199,95 \% \mathrm{CI}$ $=.15, .25$ ) did not significantly differ from the overall pooled effect size. Sensitivity analyses that excluded the lower quality study were also conducted [36], however no significant difference emerged $(r=.17,95 \% \mathrm{CI}=.11$, $.23)$.

\section{Meta-analytic results for maternal ACEs and postpartum depressive symptoms}

A total of 7 studies (5461 participants) were available for this random-effects meta-analysis, which produced a significant and positive pooled effect size of $r=.23$ (95\% CI $=.06, .39$; Fig. 2). Thus, a higher number of maternal ACEs was associated with higher reports of postpartum depressive symptoms. The funnel plot inspection was symmetrical (See Supporting Information 7) and the
Egger's test was not significant $(p=.73)$, indicating no apparent publication bias. Between study heterogeneity was indicated $\left(Q=235.95, p<.001\right.$ and $\left.I^{2}=97.46\right)$ and moderators were explored (Supporting Information 8). One significant moderator emerged: the association between postpartum depression and maternal ACEs was stronger when assessed at later postpartum timepoint. Specifically, for every 2 month unit increase in the postpartum period, the effect size increased by .04 (95\% CI: $.01, .08)$.

A sensitivity analysis was conducted without one study with a small effect size $(n=25)$ [40]. The overall pooled effect size when this study was excluded did not significantly differ from the overall pooled effect size reported $(r=.196,95 \% \mathrm{CI}=0.15, .37)$. Another sensitivity analysis examined whether excluding a study with a higher attrition level [12] would impact the pooled effect size, however there was no significant difference $(r=.256$, $95 \% \mathrm{CI}=.06, .44)$, as demonstrated by the overlapping confidence intervals. 


\section{Meta-analytic results for maternal ACEs and prenatal anxiety}

A total of 5 studies (2322 participants) were available for this random-effects meta-analysis, which produced a significant and positive pooled effect size of $r=.14$ (95\% CI $=.07, .21$; Fig. 2). Thus, a higher number of maternal ACEs was associated with higher reports of prenatal anxiety symptoms. The funnel plot inspection was symmetrical (see Supporting Information 9) and the Egger's test was not significant $(p=.99)$. Between study heterogeneity was indicated $\left(Q=11.27, p=.02\right.$ and $\left.I^{2}=64.49\right)$ and all moderators are reported in Supporting Information 10. One significant moderator emerged: the association between maternal ACEs and prenatal anxiety was highest when measured earlier in pregnancy as compared to later in pregnancy. Across studies, timing of the prenatal anxiety symptom measurement ranged from 18 to 23 weeks gestation. The meta-regression results revealed that for every 1 week unit increase in pregnancy across this range, the effect size decreased by .06 (95\% $\mathrm{CI}=-.11,-.01)$.

\section{Narrative results for maternal ACEs and postpartum anxiety}

Two studies with a total of 1235 participants examined the association between maternal ACEs and anxiety in postpartum women [18, 37]. The first study [37] found a small significant association between maternal ACEs and postpartum anxiety $(b=.16, p<.05)$, while the second study [18] did not find a significant association.

\section{Discussion}

The current study indicates that maternal ACEs are positively associated with both prenatal and postpartum depression ( $r=.19$ and $r=.23$, respectively), as well as prenatal anxiety $(r=.14)$. Overall, these results are consistent with the view that maternal ACEs are an important risk factor for poor mental health in pregnancy and postpartum period. Several processes have been proposed to explain how maternal ACEs confer a significant risk for mental health. For example, Danese and McEwen [43] describe how childhood adversity contributes to allostatic load, or wear-and-tear on the body, resulting in physiological changes to the nervous, endocrine, and immune systems. These biologically embedded changes have been hypothesized to result in greater susceptibility to mental illness when faced with psychosocial stressors [44], especially during pregnancy, when mothers face substantial neuroendocrinological [45] and psychosocial $[46,47]$ changes. Specifically, memories and cognitions regarding childhood abuse and household dysfunction may be particularly salient sources of stress during the pregnancy and transition to parenthood periods. Thus, identifying and increasing buffers of psychosocial stress (e.g., social support [48];) may be effective strategies to reduce the impact of ACEs on depression during the perinatal period.

The association between maternal ACEs and postpartum depression was stronger when measured later (up to 12 months) versus earlier in the postpartum period. Previous literature has found that postpartum depression tends to occur approximately 4 weeks after delivery [49]. However, for some women, postpartum depression may persist well beyond this timeframe and become chronic [50]. Our findings suggest that ACEs may put mothers at risk for depression across the perinatal period, which has been demonstrated to have particularly deleterious consequences for child and family outcomes [51, 52]. More research is needed to better understand the course, continuity, and mechanisms of postpartum depression in women with ACEs, as well as interventions to offset its potential chronicity. Clinicians should be aware of the possible vulnerability to chronic depression in mothers with ACEs and monitor women at risk for continued symptoms throughout the postpartum period and beyond to offset potential disease burden.

The results also suggest that exposure to childhood adversity may confer a risk of developing prenatal anxiety. Adverse childhood experiences may be associated with particular physiological changes and health risks that increase the susceptibility to anxiety $[45,53,54]$. While a paucity of research examining maternal ACEs and postnatal anxiety exists, studies have shown detrimental impacts of prenatal anxiety on child development. Specifically, prenatal anxiety may increase the risk for child socioemotional problems [8] and poorer cognitive outcomes [7]. Thus, it remains critical to examine the impact of childhood adversity on maternal mental health. Finally, moderator analyses revealed that associations among ACEs and prenatal anxiety were strongest in the first weeks of pregnancy. Thus, early identification and monitoring for anxiety in pregnancy, particularly for mothers with ACEs, may best allow for intervention to curtail future development concerns in their children.

Research on ACEs in primary care settings have signaled that the majority of women $(71.2 \%)$ report at least one childhood adversity, while a smaller number (20.5\%) report experiencing four or more of these adversities [55]. In the prenatal primary care setting, at least $46 \%$ of women report experiencing at least one childhood adversity [56]. Given the high prevalence of childhood adversity in the general population, as well as the demonstrated association between ACEs and maternal mental health identified in the current study, trauma informed approaches to prenatal and postpartum care are warranted. Trauma-informed approaches involve being informed about the impact of trauma on health and mental health, being compassionate in providing care, 
and having procedures and policies that resist causing distress to individuals who may have a trauma history. In some instances, asking about an individual's trauma history may be relevant for their medical care, but in other instances it may not [17]. Prioritizing traumainformed approaches to care is more important than simply providing an ACEs questionnaire to ask about childhood adversity. It is also critical for healthcare providers to consider cumulative adversity that may have occurred across the lifecourse and how this may influence maternal mental health.

\section{Limitations}

Several limitations of the current meta-analytic study should be noted. First, assessment of maternal ACEs was based on retrospective self-report. Recall of ACEs history may be susceptible to memory inaccuracies which may be especially compromised among participants who have experienced trauma [57]. We did not include other retrospective measures of child maltreatment or adversity in the current study in order to obtain more homogeneous estimates of the association between the cumulative nature of ACEs and maternal mental health. Future meta-analyses may consider broadening the search criteria to include discrete adversity items (e.g., physical abuse or sexual abuse), as well as other measures of childhood maltreatment and trauma. Second, analyses are based on reports of depressive and anxiety symptoms, not diagnoses of disorders. It will be important in future research to determine if maternal ACEs increase risk for mood or anxiety disorders in the perinatal period. Third, the present study could only narratively describe the association between maternal ACEs and postpartum anxiety due to a small number of available studies. This represents an important area for future research given the heterogeneous findings of the presently included studies. Fourth, no protocol was registered for the current review as it was part of a large series of meta-analyses that were conducted on ACEs. Finally, the majority of participants in the included studies resided in North America, and as such, these results may be limited in terms of their generalizability across cultures and countries outside of North America.

\section{Conclusions}

In sum, this study sheds light on the associations between maternal ACEs and subsequent mental health difficulties during pregnancy and the postpartum period. Specifically, our findings suggest that ACEs are likely an important determinant of maternal mental health difficulties, particularly prenatal and postpartum depression, which may in turn put infants at risk for physical and mental health difficulties $[3,7,8]$. In order to mitigate risk, pre- and postpartum mental health screening are imperative, particularly for women where a known trauma history is identified. Trauma-informed approaches to patient care, as well as increased support during and after pregnancy, are warranted to offset the relative risk of ACEs on maternal mental health.

\section{Supplementary Information}

The online version contains supplementary material available at https://doi. org/10.1186/s12888-020-03017-w.

\section{Additional file 1.}

\section{Abbreviations}

ACEs: Adverse Childhood Experiences

\section{Acknowledgements}

The authors acknowledge Cheri Nickel, MLIS (University of Calgary), for conducting the literature search for this project.

\section{Authors' contributions}

NR and SM conceptualized the project and designed the search. NR, CD, JC, $R E$, JZ conducted the abstract review and data extraction. NR and SM conducted the data analysis. NR, CD, JC, RE, JZ, and SM drafted the manuscript and critically reviewed it prior to submission. All authors have read and approved the manuscript.

\section{Funding}

Funding support was provided by the Canada Research Chairs Program (SM), Alberta Innovates (NR, JC), and the Social Sciences and Humanities Research Council of Canada (RE). The funders had no role in the design of the study, data collection, data analysis, interpretation of the data, or writing of the manuscript.

\section{Availability of data and materials}

The data used for this meta-analysis are publicly available in the research studies. The full dataset can be requested from the corresponding author on reasonable request.

Ethics approval and consent to participate

Ethics was not obtained as this is a review of existing literature.

\section{Consent for publication}

N/A.

\section{Competing interests}

There are no competing interests to report.

Received: 13 September 2020 Accepted: 17 December 2020

Published online: 11 January 2021

\section{References}

1. Gelaye B, Rondon MB, Araya R, Williams MA. Epidemiology of maternal depression, risk factors, and child outcomes in low-income and middleincome countries. Lancet Psychiatry. 2016;3(10):973-82.

2. WHO: Maternal mental health and child health and development in low and middle income countries. 2008.

3. Slomian J, Honvo G, Emonts P, Reginster JY, Bruyere O. Consequences of maternal postpartum depression: a systematic review of maternal and infant outcomes. Womens Health. 2019;15:1745506519844044.

4. Alder J, Fink N, Bitzer J, Hosli I, Holzgreve W. Depression and anxiety during pregnancy: a risk factor for obstetric, fetal and neonatal outcome? A critical review of the literature. J Matern Fetal Neonatal Med. 2007;20(3):189-209.

5. Horrigan TJ, Schroeder AV, Schaffer RM. The triad of substance abuse, violence, and depression are interrelated in pregnancy. J Subst Abus Treat. 2000;18(1):55-8.

6. Szekely E, Neumann A, Sallis H, Jolicoeur-Martineau A, Verhulst FC, Meaney MJ, Pearson RM, Levitan RD, Kennedy JL, Lydon JE, et al. Maternal prenatal mood, pregnancy-specific worries, and early child psychopathology: 
findings from the DREAM BIG consortium. J Am Acad Child Adolesc Psychiatry. 2021;60(1):186-197.

7. Tarabulsy GM, Pearson J, Vaillancourt-Morel MP, Bussieres EL, Madigan S, Lemelin JP, Duchesneau AA, Hatier DE, Royer F. Meta-analytic findings of the relation between maternal prenatal stress and anxiety and child cognitive outcome. J Dev Behav Pediatr. 2014;35(1):38-43.

8. Madigan S, Oatley H, Racine N, Fearon RMP, Schumacher L, Akbari E, Cooke JE, Tarabulsy GM. A meta-analysis of maternal prenatal depression and anxiety on child Socioemotional development. J Am Acad Child Adolesc Psychiatry. 2018;57(9):645-657 e648.

9. Grote NK, Bridge JA, Gavin AR, Melville JL, lyengar S, Katon WJ. A metaanalysis of depression during pregnancy and the risk of preterm birth, low birth weight, and intrauterine growth restriction. Arch Gen Psychiatry. 2010; 67(10):1012-24.

10. Abel KM, Hope H, Swift E, Parisi R, Ashcroft DM, Kosidou K, Osam CS, Dalman C, Pierce M. Prevalence of maternal mental illness among children and adolescents in the UK between 2005 and 2017: a national retrospective cohort analysis. Lancet Public Health. 2019;4(6):e291-300.

11. Bennett HA, Einarson A, Taddio A, Koren G, Einarson TR. Prevalence of depression during pregnancy: systematic review. Obstet Gynecol. 2004; 103(4):698-709.

12. Racine N, Zumwalt K, McDonald S, Tough S, Madigan S. Perinatal depression: the role of maternal adverse childhood experiences and social support. J Affect Disord. 2020;263:576-81.

13. Wajid A, van Zanten SV, Mughal MK, Biringer A, Austin MP, Vermeyden L, Kingston D. Adversity in childhood and depression in pregnancy. Arch Womens Ment Health. 2020;23(2):169-80.

14. Buss C, Entringer S, Moog NK, Toepfer P, Fair DA, Simhan HN, Heim CM, Wadhwa PD. Intergenerational transmission of maternal childhood maltreatment exposure: implications for fetal brain development. J Am Acad Child Adolesc Psychiatry. 2017;56(5):373-82.

15. Baumeister $\mathrm{D}$, Lightman SL, Pariante CM. The interface of stress and the HPA axis in behavioural phenotypes of mental illness. Curr Top Behav Neurosci. 2014;18:13-24.

16. Hudziak JJ. ACEs and pregnancy: Time to support all expectant mothers. Pediatrics. 2018;141(4):e20180232

17. Racine N, Killam T, Madigan S. Trauma-informed care as a universal precaution: beyond the adverse childhood experiences questionnaire. JAMA Pediatr. 2019;174:1-2.

18. Menke R, Swanson L, Erickson N, Reglan G, Thompson S, Bullard K, Rosenblum K, Lopez J, Muzik M. Childhood adversity and sleep are associated with symptom severity in perinatal women presenting for psychiatric care. Arch Womens Ment Health. 2018;22(4):457-65.

19. Putnam KT, Wilcox M, Robertson-Blackmore E, Sharkey K, Bergink V, MunkOlsen T, Deligiannidis KM, Payne J, Altemus M, Newport J, Apter G, Devouche E, Viktorin A, Magnusson P, Penninx B, Buist A, Bilszta J, O'Hara M, Stuart S, Brock R, Roza S, Tiemeier H, Guille C, Epperson N, Kim D, Schmidt P, Martinez P, Di Florio A, Wisner KL, Stowe Z, Jones I, Sullivan PF, Rubinow D, Wildenhaus K, Meltzer-Brody S. Clinical phenotypes of perinatal depression and time of symptom onset: analysis of data from an international consortium. Lancet Psychiatry. 2017;4(6):477-85.

20. Kramer MS, Seguin L, Lydon J, Goulet L. Socio-economic disparities in pregnancy outcome: why do the poor fare so poorly? Paediatr Perinat Epidemiol. 2000;14(3):194-210.

21. Bussieres EL, Tarabulsy GM, Pearson J, Tessier R, Forest J, Giguere Y. Maternal prenatal stress and infant birth weight and gestational age: a meta-analysis of prospective studies. Dev Rev. 2015:179-99.

22. Angerud K, Annerback E-M, Tyden T, Boddeti S, Kristiansson P. Adverse childhood experiences and depressive symptomatology among pregnant women. Acta Obstet Gynecol Scand. 2018;97(6):701-8.

23. Felitti VJ, Anda RF, Nordenberg D. Relationship of childhood abuse and household dysfunction to many of the leading causes of death in adults: the adverse childhood experiences (ACE) study. Am J Prev Med. 1998;14(4): 245-58

24. Moher D, Liberati A, Tetzlaff J, Altman DG, Group TP. Preferred reporting items for systematic reviews and meta-analyses: the PRISMA statement. PLoS Med. 2009;6(7):e1000097.

25. Voils $\mathrm{Cl}$, Crandell JL, Chang Y, Leeman J, Sandelowski M. Combining adjusted and unadjusted findings in mixed research synthesis. J Eval Clin Pract. 2011:17(3):429-34.
26. Borenstein $M$, Hedges L, Higgins J, Rothstein H. Comprehensive metaanalysis version 3. Englewood: Biostat; 2013.

27. Rosenthal R. Writing meta-analytic reviews. Psychol Bull. 1995;118(2):183

28. Funder DC, Ozer DJ. Evaluating effect science in psychological research: sense and nonsense. Adv Methods Pract Psychol Sci. 2019;2(2):156-68.

29. Borenstein M, Hesdges L, Higgins J, Rothstein H. Introduction to metaanalysis. West Sussex: Wiley; 2009.

30. Harrer M, Cuijpers P, Furukawa TA, Ebert DD. Doing Meta-Analysis in R: A Hands-on Guide; 2019.

31. Thompson SG, Higgins JP. How should meta-regression analyses be undertaken and interpreted? Stat Med. 2002;21(11):1559-73.

32. Appleton A, Kevin K, Holdsworth E, Schell L. Social support during pregnancy modifies the association between maternal adverse childhood experiences and infant birth size. Matern Child Health J. 2019;23(3):408-15.

33. Folger AT, Eismann EA, Stephenson NB, Shapiro RA, Macaluso M, Brownrigg $M E$, Gillespie RJ. Parental adverse childhood experiences and offspring development at 2 years of age. Pediatrics. 2018;141(4):e20172826.

34. Fredriksen E, Von Soest $T$, Smith L, Moe V. Patterns of pregnancy and postpartum depressive symptoms: latent class trajectories and predictors. J Abnorm Psychol. 2017;126(2):173-83.

35. Hantsoo L, Jašarević E, Criniti S, McGeehan B, Tanes C, Sammel MD, Elovitz MA, Compher C, Wu G, Epperson CN. Childhood adversity impact on gut microbiota and inflammatory response to stress during pregnancy. Brain Behav Immun. 2019;75:240-50.

36. Howell KH, Miller-Graff LE, Schaefer LM, Scrafford KE. Relational resilience as a potential mediator between adverse childhood experiences and prenatal depression. J Health Psychol. 2017;25(4):545-57.

37. Letourneau N, Dewey D, Kaplan BJ, Ntanda H, Novick J, Thomas JC, Deane AJ, Leung B, Pon K, Giesbrecht GF. Intergenerational transmission of adverse childhood experiences via maternal depression and anxiety and moderation by child sex. J Dev Orig Health Dis. 2019;10(1):88-99.

38. Mersky JP, Janczewski CE. Adverse childhood experiences and postpartum depression in home visiting programs: prevalence, association, and mediating mechanisms. Matern Child Health J. 2018;22(7):1051-8.

39. Miller-Graff $L E$, Ahmed AH, Paulson JL. Intimate partner violence and breastfeeding outcomes in a sample of low-income women. J Hum Lact. 2018;34(3):494-502.

40. Morrison KE, Epperson CN, Sammel MD, Ewing G, Podcasy JS, Hantsoo L, Kim DR, Bale TL. Preadolescent adversity programs a disrupted maternal stress reactivity in humans and mice. Biol Psychiatry. 2017;81(8):693-701.

41. Narayan AJ, Rivera LM, Bernstein RE, Harris WW, Lieberman AF. Positive childhood experiences predict less psychopathology and stress in pregnant women with childhood adversity: A pilot study of the benevolent childhood experiences (BCEs) scale. Child Abuse Negl. 2018;78:19-30.

42. Young-Wolff KC, Alabaster A, McCaw B, Stoller N, Watson C, Sterling S, Ridout KK, Flanagan T. Adverse childhood experiences and mental and behavioral health conditions during pregnancy:the role of resilience. J Womens Health. 2019;28(4):452-62.

43. Danese A, McEwen BS. Adverse childhood experiences, allostasis, allostatic load, and age-related disease. Physiol Behav. 2012;106(1):29-39.

44. Hammen C, Henry R, Daley SE. Depression and sensitization to stressors among young women as a function of childhood adversity. J Consult Clin Psychol. 2000;68(5):782-7.

45. Feldman R, Weller A, Zagoory-Sharon O, Levine A. Evidence for a neuroendocrinological foundation of human affiliation: plasma oxytocin levels across pregnancy and the postpartum period predict mother-infant bonding. Psychol Sci. 2007;18(11):965-70.

46. Christie H, Talmon A, Schäfer SK, de Haan A, Louison Vang M, Gilbar O, Alisic E, Brown E. The transition to parenthood following a history of childhood maltreatment: a review of the literature on prospective and new parents' experiences. Eur J Psychotraumatol. 2017:8:1492834.

47. Cowan CP, Cowan PA. Interventions to ease the transition to parenthood: why they are needed and what they can do. Fam Relat. 1995;44(4):412-23.

48. Racine N, Madigan S, Plamondon A, Hetherington E, McDonald S, Tough S. Maternal adverse childhood experiences and antepartum risks: the moderating role of social support. Arch Womens Ment Health. 2018;21(6):663-70.

49. Hendrick V, Altshuler L, Strouse T, Grosser S. Postpartum and nonpostpartum depression: difference in presentation and response to pharmacologic treatment. Depress Anxiety. 2000;11(2):66-72.

50. Vliegen N, Casalin S, Luyten P. The course of postpartum depression: a review of longitudinal studies. Harvard Rev Psychiatry. 2014;22(1):1-22. 
51. Grace SL, Evindar A, Stewart DE. The effect of postpartum depression on child cognitive development and behavior: a review and critical analysis of the literature. Arch Womens Ment Health. 2003;6:263-74.

52. Hentges RF, Graham SA, Fearon P, Tough S, Madigan S. The chronicity and timing of prenatal and antenatal maternal depression and anxiety on child outcomes at age 5. Depressi Anxiety. 2020;37(6):576-86.

53. Choi KW, Sikkema KJ. Childhood maltreatment and perinatal mood and anxiety disorders: a systematic review. Trauma Violence Abuse. 2016;17(5):427-53.

54. Christian LM, Franco A, Glaser R, lams JD. Depressive symptoms are associated with elevated serum proinflammatory cytokines among pregnant women. Brain Behav Immun. 2009;23(6):750-4.

55. Dobson K, Pusch D, Allan L, Gonzalez S, Poole J, Marandola G. The long shadow of adverse childhood events: physical health outcomes in an adult community sample. Am J Prevent Med Pub Health. 2020;6(2):39-49.

56. Flanagan T, Alabaster A, McCaw B, Stoller N, Watson C, Young-Wolff KC. Feasibility and acceptability of screening for adverse childhood experiences in prenatal care. J Women's Health (Larchmt). 2018;27(7):903-11.

57. Danby MC, Brubacher SP, Sharman SJ, Powell MB, Roberts KP. Children's reasoning about which episode of a repeated event is best remembered. Appl Cogn Psychol. 2017;31:99-108.

\section{Publisher's Note}

Springer Nature remains neutral with regard to jurisdictional claims in published maps and institutional affiliations.

Ready to submit your research? Choose BMC and benefit from:

- fast, convenient online submission

- thorough peer review by experienced researchers in your field

- rapid publication on acceptance

- support for research data, including large and complex data types

- gold Open Access which fosters wider collaboration and increased citations

- maximum visibility for your research: over $100 \mathrm{M}$ website views per year

At BMC, research is always in progress.

Learn more biomedcentral.com/submissions 